\title{
Complete nontuberculous mycobacteria whole genomes using an optimized DNA extraction protocol for long-read sequencing
}

\author{
Jennifer M. Bouso ${ }^{1}$ and Paul J. Planet ${ }^{2,3,4^{*}}$ (i)
}

\begin{abstract}
Background: Nontuberculous mycobacteria (NTM) are a major cause of pulmonary and systemic disease in at-risk populations. Gaps in knowledge about transmission patterns, evolution, and pathogenicity during infection have prompted a recent surge in genomic NTM research. Increased availability and affordability of whole genome sequencing (WGS) techniques provide new opportunities to sequence and construct complete bacterial genomes faster and at a lower cost. However, extracting large quantities of pure genomic DNA is particularly challenging with NTM due to its slow growth and recalcitrant cell wall. Here we report a DNA extraction protocol that is optimized for long-read WGS of NTM, yielding large quantities of highly pure DNA with no additional clean-up steps.

Results: Our DNA extraction method was compared to 6 other methods with variations in timing of mechanical disruption and enzymatic digestion of the cell wall, quantity of matrix material, and reagents used in extraction and precipitation. We tested our optimized method on 38 clinical isolates from the M. avium and M. abscessus complexes, which yielded optimal quality and quantity measurements for Oxford Nanopore Technologies sequencing. We also present the efficient completion of circularized $M$. avium subspecies hominissuis genomes using our extraction technique and the long-read sequencing MinlON platform, including the identification of a novel plasmid.

Conclusions: Our optimized extraction protocol and assembly pipeline was both sufficient and efficient for genome closure. We expect that our finely-tuned extraction method will prove to be a valuable tool in long-read sequencing and completion of mycobacterial genomes going forward. Utilization of comprehensive, long-read based approaches will advance the understanding evolution and pathogenicity of NTM infections.

Keywords: Mycobacteria, Long-read sequencing, Whole genome sequencing, Nontuberculous mycobacteria, Mycobacterium avium complex, Mycobacterium abscessus complex, Genome assembly, Cystic fibrosis, Chronic obstructive pulmonary disease, Bronchiectasis
\end{abstract}

\section{Background}

The emergence of nontuberculous mycobacteria (NTM) infection in immunocompromised hosts, the elderly, patients with cystic fibrosis $(\mathrm{CF})$, and patients with non-CF chronic lung disease (COPD, asthma, non-CF bronchiectasis) has prompted genomic investigations aimed at

\footnotetext{
* Correspondence: planetp@email.chop.edu

${ }^{2}$ Division of Infectious Diseases, Children's Hospital of Philadelphia,

Philadelphia, PA, USA

${ }^{3}$ Perelman School of Medicine, University of Pennsylvania, Philadelphia, PA, USA

Full list of author information is available at the end of the article
}

uncovering the determinants of pathogenicity, transmission, evolution, and adaptation [1-10]. Bacterial evolution and phylogenomic research have been revolutionized by more available and affordable whole genome sequencing (WGS) [11-15]. WGS of NTM has begun to shed light on taxonomic conundrums, transmissibility, and global evolution [16-24]. However, the unique challenges of slow growth rates and inefficient DNA extraction have impeded rigorous genomic investigation of NTM.

Over recent years, the vast majority of genomic analyses have relied on short-read, shot-gun sequencing

(c) The Author(s). 2019 Open Access This article is distributed under the terms of the Creative Commons Attribution 4.0 International License (http://creativecommons.org/licenses/by/4.0/), which permits unrestricted use, distribution, and 
(75-500 base pairs), which can deliver exceptional accuracy, but rarely produce closed genomes. Indeed, less than $10 \%$ of available microbial genomes are complete [25]. During comparative analyses, fragmented assemblies are problematic because they may unlink gene clusters, fail to resolve repetitive and $\mathrm{G}+\mathrm{C}$ rich regions, neglect insertion and deletion elements (indels), and overlook recombination [26-29].

Long-read sequencing promises an enhanced ability to complete bacterial genomes. The most commonly available techniques for long-read sequencing are the Single Molecule Real-Time (SMRT) technology by Pacific Biosciences $^{\bullet}$ (PacBio, United States) and the newer Oxford Nanopore Technologies (ONT, United Kingdom) MinION $[14,27,30]$. Unlike most short-read sequencing methods, which require only very small amounts of DNA (as low as $1 \mathrm{ng}$ ), long-read platforms require high quantities of very pure DNA for acceptable processing. DNA purity and integrity (i.e., length or molecular weight [MW]) is not only essential for functionality of the sequencer, but also is directly related to the quality of downstream bioinformatic analyses, as the DNA MW places a natural upper bound on the potential read length. ONT MinION sequencing requires input of 400-1000 ng of high MW DNA (average fragment size of $>30 \mathrm{~kb}$ ) with low solvent/salt and protein contamination (optical density [OD] 260/230 2.0-2.2 and 260/280 1.8, respectively) (nanoporetech.com).

Extracting large quantities of intact, pure genomic DNA is exceptionally challenging with NTM due to their hardy, lipid-laden mycobacterial cell wall. Standard extraction techniques (i.e., commercial kits) do not yield sufficient quantities of DNA for WGS while overly vigorous techniques shear DNA into suboptimal MWs for long-read sequencing. In our experience, the standard protocols specific for mycobacterial DNA extraction were unable to yield DNA that was of sufficient quality for ONT MinION sequencing [31-36]. We thus developed an optimized protocol over the course of performing hundreds of NTM DNA extractions using components of several extraction techniques, initially through trial and error, and subsequently confirmed by direct comparisons as described here. Our optimized method varies from the widely-used Käser et al. method by early bead-beating (prior to enzymatic digestion, as opposed to after) in high concentrations of sodium dodecyl sulfate (SDS) followed by gentle gel-based extraction to protect long strands of DNA, and an isopropanol precipitation favoring DNA purification above DNA quantity.

The goal of developing our protocol was to extract large amounts of high MW, pure DNA for use in longread WGS. To evaluate the subtle alterations in methodology, we compared 6 variations in design, demonstrate the superiority of our optimized technique, validate its use on a large number of clinical isolates of two NTM species complexes, and prove its capacity for producing sufficient reads by the ONT MinION sequencer for genome completion. We also present three complete and circularized genomes constructed with ONT reads as well as a novel plasmid.

\section{Results \\ Method comparison, validation, sequencing, and assembly Methods defined}

Our full DNA extraction protocol can be found in Additional file 1. An experiment was designed to test 6 variables in a standard phenol-based extraction technique; variable choices were made based on a number of grid experiments completed previously (Additional file 2: Table S1). Alterations in methodology included the timing of mechanical disruption, the quantity of beads used for mechanical disruption, extraction with phenol versus chloroform-isoamyl alcohol only, precipitation with either cold ethanol or room temperature isopropanol, sodium chloride versus sodium acetate precipitation, and the number of final washes. Variations in methods are outlined in Table 1 . Notably, all methods were performed in a "Total Lysis Buffer" (TLB) that was previously found to be superior in direct comparison to a standard buffer (Additional file 2: Table S1); see Additional file 1 for TLB composition. Method 3 is most similar to a standard protocol by Käser et al. [32]. Method 5 is our optimized method and was the only method to produce sufficient quantity and quality standards for the ONT MinION long-read sequencer.

\section{Method comparison}

Bacterial pellets averaged a normalized "washed weight" of $26.4 \mathrm{mg}$. With the exception of Method 6, all methods produced sufficient total DNA quantity (Fig. 1A). Method 1 produced the highest total amounts of DNA (mean $12.45 \mu \mathrm{g}$, standard deviation [SD] 2.928). All methods with the exception of Method 6 gave sufficient 260/280, indicating low protein contamination overall (Fig. 1B). Method 3 and 5 produced the highest 260/280 measurements, which were significantly higher than other methods (Fig. 1B). Only Method 5 produced sufficient 260/230 for use with longread sequencers, which was significantly higher than all other methods (Fig. 1C). Despite variations in quantity, all methods produced high MW DNA as evidenced on an agarose gel, indicating preservation of high MW fragments of genomic DNA (Fig. 1D). For deeper comparison, Method 1 and Method 5 DNA extractions were also analyzed on a bioanalyzer (Fig. 1E) with peak means of 29,369 base pairs (SD 20,002 bp) and 51,598 base pairs (SD 5,882 bp), respectively. While there was a trend toward higher MW DNA fragments achieved by Method 5, the differences were not significant (paired t-test, $p=0.1450$ ). In short, 
Table 1 Differentiation of tested methods by variable

\begin{tabular}{|c|c|c|c|c|c|c|c|}
\hline & Method 1 & Method 2 & Method 3 & Method 4 & Method 5 & Method 6 & Method 7 \\
\hline 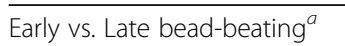 & Early & Late & Late & Early & Early & Early & Early \\
\hline Bead Quantity & $150 \mathrm{mg}$ & $150 \mathrm{mg}$ & $150 \mathrm{mg}$ & $75 \mathrm{mg}$ & $150 \mathrm{mg}$ & $150 \mathrm{mg}$ & $150 \mathrm{mg}$ \\
\hline Phenol vs. No Phenol ${ }^{b}$ & No phenol & No phenol & Phenol & No phenol & Phenol & No phenol & No phenol \\
\hline Precipitation Temp/Reagent ${ }^{c}$ & RT/2-Prop & RT/2-Prop & Cold ETOH & RT/2-Prop & RT/2-Prop & Cold ETOH & RT/2-Prop \\
\hline Precipitation Salt ${ }^{d}$ & $\mathrm{NaOAC}$ & $\mathrm{NaOAC}$ & $\mathrm{NaOAC}$ & $\mathrm{NaOAC}$ & $\mathrm{NaCl}$ & $\mathrm{NaOAC}$ & $\mathrm{NaOAC}$ \\
\hline Number of washes & 3 & 3 & 3 & 3 & 3 & 3 & 5 \\
\hline
\end{tabular}

a "Early" bead-beating refers to the timing prior to enzymatic digestion; "Late" bead-beating refers to timing after enzymatic digestion All Early bead-beating was done in high SDS concentration, see Additional file 1

b DNA extractions in "no phenol" were extracted as described in Methods with chloroform:isoamyl alcohol (24:1, Tris-saturated)

Extractions in "phenol" were extracted using phenol:chloroform:isoamyl alcohol (25:24:1, Tris-saturated, pH 8.0)

c Precipitation reagent was either RT 2-Prop (room temperature isopropanol) or Cold ETOH (ethanol). See Additional file 4: Figure S1

${ }^{d}$ Precipitation salt was either $3 \mathrm{M}$ sodium acetate $(\mathrm{pH} 5.2)$ or $5 \mathrm{M} \mathrm{NaCl}$

Method 5 was the only method to produce sufficiently pure DNA for ONT MinION sequencing without requiring additional clean-up steps in all quality and quantity measurements with mean total DNA of $7.263 \mu \mathrm{g}$ (SD 0.50), mean $260 / 280$ of 1.893 (SD 0.012), and mean 260/230 of 1.947 (SD 0.025).

\section{Method validation}

Of 38 clinical isolates from 8 patients extracted with our optimized method (Method 5), 12 isolates were identified as Mycobacterium avium complex (MAC, slow-growing), and 26 isolates were Mycobacterium abscessus complex (MABSC, rapid-growing) by taxonomic classification. Notably, all 38 extractions using Method 5 yielded sufficient quality and quantity measurements for long-read sequencing without requiring any additional clean-up steps (Fig. 2a-c). All DNA extracts achieved high enough quantity of DNA (mean $4.17 \mu \mathrm{g}$, SD 0.80 ) and quality of DNA with mean 260/230 of 2.29 (SD 0.33) and mean 260/280 of 1.88 (SD 0.069), meeting the required specifications by

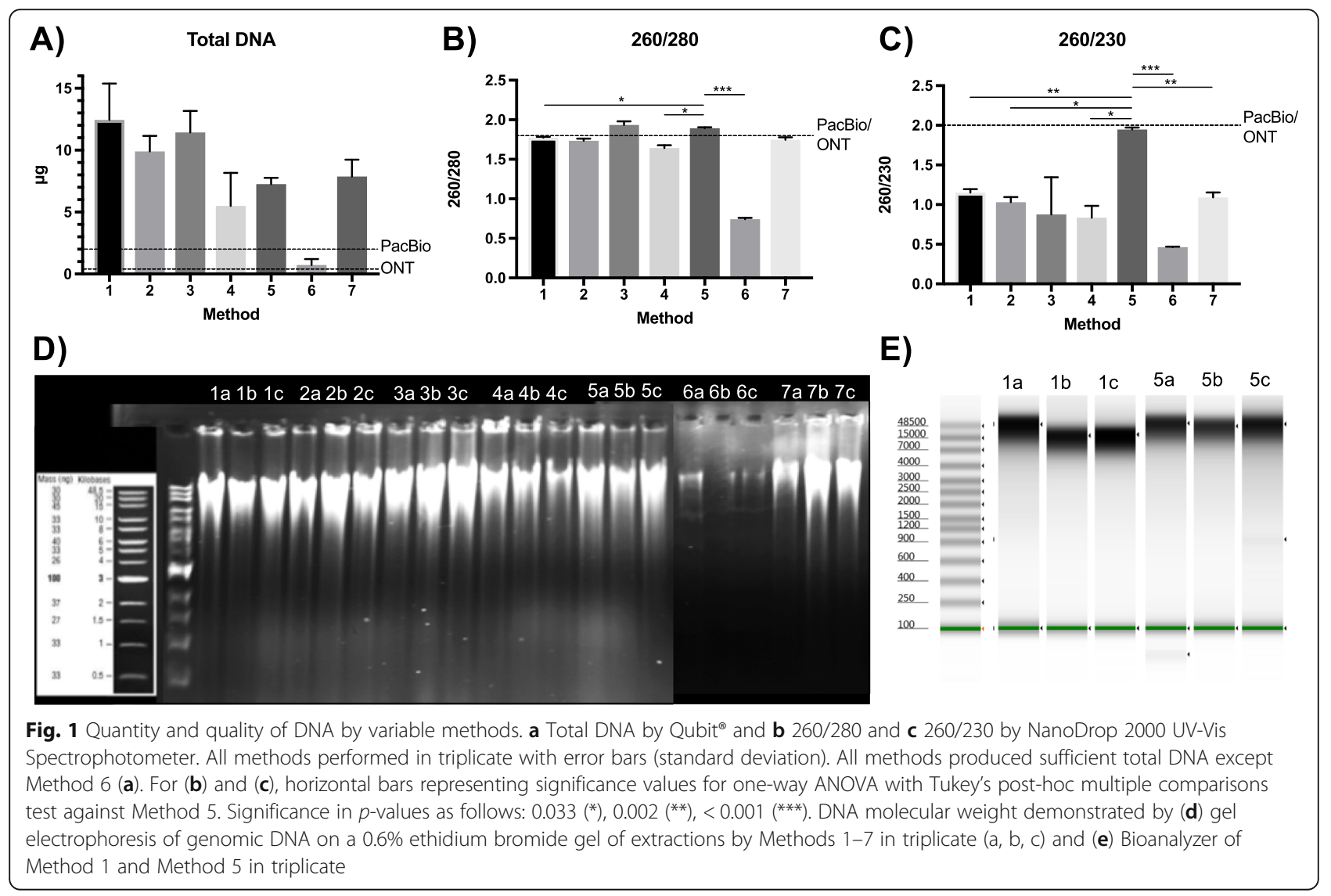




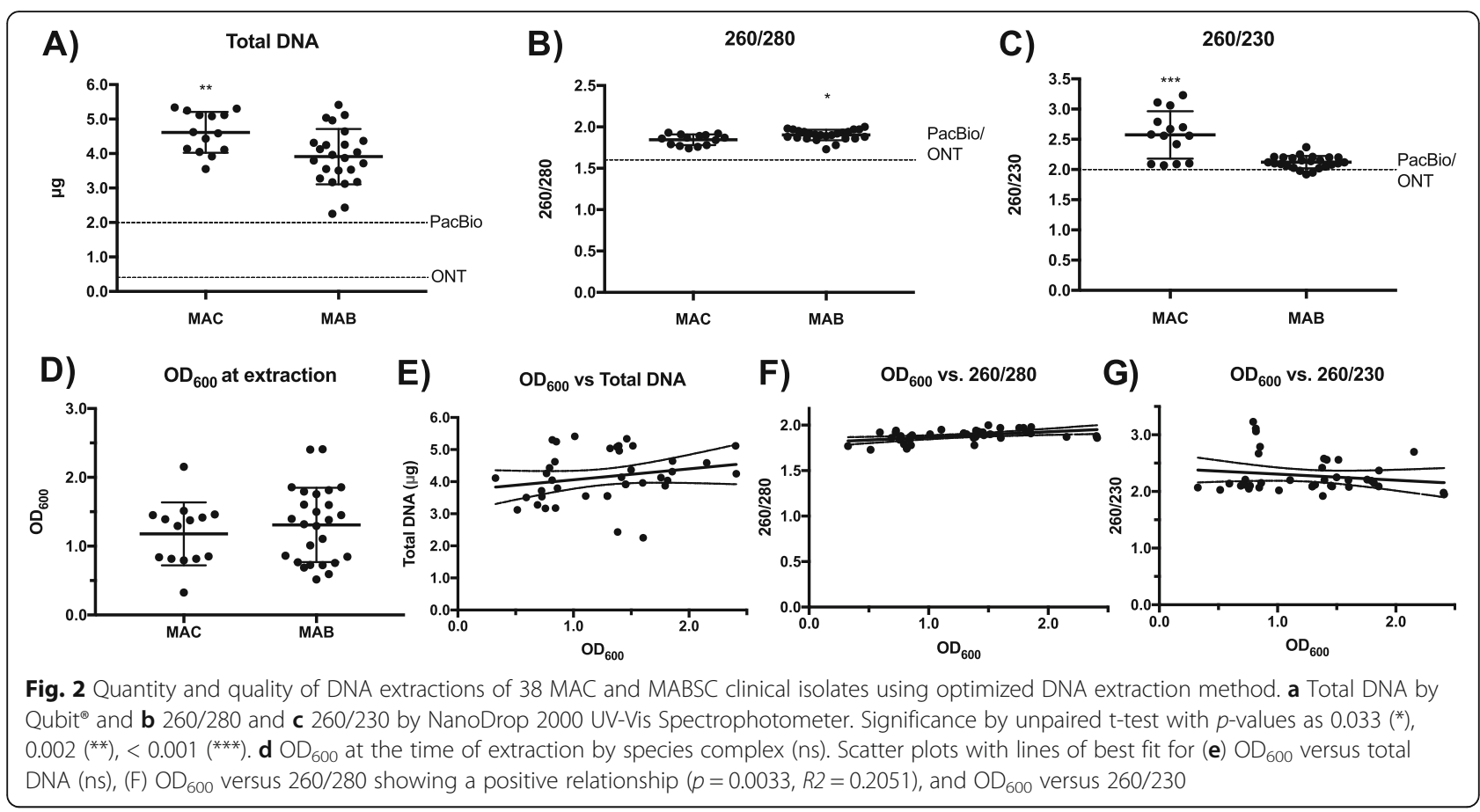

the ONT manufacturer. Although all DNA extractions were adequate for ONT sequencing, we also found that MABSC samples had significantly higher 260/280 ( $p=$ $0.01)$ and total DNA $(p=0.007)$, while MAC samples had significantly higher 260/230 ( $p<0.001)$ (Fig. 2a-c). Due to wide variation in starting $\mathrm{OD}_{600}$ among NTMs ([0.3302.409 ]; mean $=1.225$, median $=1.294$ ), we also investigated if $\mathrm{OD}_{600}$ correlated with extraction outcomes among all MAC and MABSC samples. While $\mathrm{OD}_{600}$ between MAC and MABSC samples did not vary significantly at the time of extraction (Fig. 2d), we observed a positive relationship between $\mathrm{OD}_{600}$ and $260 / 280$ for all samples $\left(p=0.0033, R^{2}=0.2051\right)$ (Fig. $\left.2 \mathrm{f}\right)$. No significant relationship was found between $\mathrm{OD}_{600}$ and total DNA or 260/230 (Fig. 2e, g).

\section{ONT whole genome sequencing and assembly}

Three isolates from MAC single colonies (CHOP101034, CHOP101115, and CHOP101174) were chosen for longread sequencing as biological replicates. As Method 5 was the only method to produce sufficient quantity and quality of DNA for long-read sequencing, use of an alternative method for comparison required additional clean-up steps (see Additional file 1 for isopropanol clean-up steps). As clean-up of DNA inevitably results in significant loses of total DNA, we chose Method 1 for comparison, as it yielded the highest total DNA. All DNA extracts achieved high enough quantity and quality prior to ONT sequencing. Of note, the ONT MinION sequencer is very sensitive to poor-quality DNA, and samples with low 260/230 will cause osmotic imbalances in the flow cell (Experiment protocol, SQK-RBK-004, ONT).

Despite having sufficient quantity and quality of DNA and using the same library preparation and parameters for both run preparations, the Method 5 sequencing run gave superior total reads and total bases sequenced with significantly higher mean $(p=0.0168)$ and median $(p=$ 0.0101 ) read lengths per barcode (Table 2). To assess for variability between MAC and MAB sequencing, we additionally completed WGS on 8 isolates from 8 different patients selected from the 38 clinical samples mentioned previously. Extraction and sequencing data for this 8sample run can be found in Additional file 3: Table S2. There were no significant differences between MAC and $M A B$ sequencing results with regard to total reads, total bases, mean or median read lengths, or longest read sequenced (unpaired t-tests). Plots of sequencing run outputs for all ONT MinION runs are displayed in Additional file 4: Figure S1. A complete list of all strains extracted by the optimized extraction method, Method 5 , is also available (Additional file 5: Table S4).

\section{Complete genomes}

The final, long-read based MAC genome assemblies were complete or near-complete with mean genome size $5.316 \mathrm{Mb}$ and $69.01 \%$ GC content (Table 3). Finished genome assemblies compared between two investigated methods of DNA extraction (Method 1 with isopropanol clean-up versus method 5 without clean-up) did not vary significantly by statistical analyses (paired t-tests) with regard to length, contig number, N50, \%GC, or coverage 
Table 2 ONT Sequencing Run Statistics

\begin{tabular}{llllllll}
\hline & Total reads & Total bases & Mean read length (bp) & Median read length (bp) & Longest read & Phred score & \%Error Probability \\
\hline Method 1 & $1,455,977$ & $1,634,706,997$ & 1122.8 & 751.0 & 34,548 & 13 & $5.011 \%$ \\
Method 5 & $1,500,966$ & $2,778,573,523$ & $1851.2^{*}$ & $1196.0^{*}$ & 64,327 & 14 & $3.981 \%$
\end{tabular}

Raw values are listed for each MinION ONT runs using DNA extracted by Method 1 (after additional clean-up) and by Method 5. For statistical analyses comparing Method $1(n=3)$ versus Method $5(n=3)$, paired-t test comparisons (total reads, total bases, mean read length, median read length, longest read) were completed on basecalled, demultiplexed, and trimmed reads. Mean read length and median read length were significantly longer from Method $5, p=0.0168$ and $p=0.0101$, respectively. Notably, Phred score was higher and error probability lower in the Method 5 run, which may reflect higher quality substrates

* Indicates $p$-value of $p<0.05$

${ }^{\mathrm{a}}$ Error probability percentage is a function of Mean Phred score, where probability $\mathrm{P} \%=100^{*} 10 \wedge(-\mathrm{Phred} / 10)$

(Table 3). As the same short-reads were used for polishing both Method 1 and Method 5 assemblies, direct comparisons were able to be completed without variability introduced by short-read data (Additional file 6: Table S3). All three Method 5 assemblies were complete and circularized, while only $1 / 3$ of Method 1 bacterial chromosomes were complete and circularized. While not reaching statistical significance, Method 5 genomes had lower overall contamination scores with means of 0.9 (Method 5, SD 0) versus 3.3 (Method 1, SD 2.12), $p=0.1210$. Method 5 genomes had significantly higher fine consistency with a mean of 97.37 (Method 5, SD 0.116 ) versus 96.2 (Method 1, SD 0.608), $p=0.0310$.

The cost for completing each genome was approximately $\$ 280$ US dollars. This could be reduced to as low as $\$ 80$ per genome by including more barcodes in the sequencing run; however, in our experience, increasing barcodes decreases coverage per genome (Additional file 3: Table S2). In summary, our optimized protocol for long-read DNA extraction and our assembly pipeline has allowed us to produce DNA sufficient for long-read sequencing after a single extraction without additional clean-up steps, and furthermore, allows us to present the first publicly-available $M$. avium subspecies hominissuis genome assemblies constructed utilizing ONT longreads.
We present three genome assemblies: CHOP101034, CHOP101115, and CHOP101174, which were all isolated from an adolescent with $\mathrm{CF}$ and chronic MAC infection between 2016 and 2017 (Fig. 3). All genomes were identified as $M$. avium subspecies hominissuis and are considered clonal isolates by core whole genome alignment (data not shown). Genomic DNA was extracted by Method 5 and sequenced, assembled, and annotated as described above. Two plasmids were identified in the assemblies, including a novel plasmid that we designate here as pMARIA (plasmid Mycobacterium avium Replicon [class] 1 [type] a), and a plasmid previously described by Caverly et al., pFLAC0181 (GenBank: CP023150.1, BioSample SAMN07528789, unpublished) identified by WGS from an isolate of $M$. intracellularae.

CHOP101034 consists of a complete, circularized chromosome and two circularized plasmids. CHOP101034 has 5,368,111 base pairs with $68.93 \%$ GC content, 5, 216 coding sequences (CDS), 99 repeat regions, 47 tRNAs, 3 rRNAs, and mean long-read ONT coverage of $83.12 x$. The novel plasmid identified, pMARIA, is 41,578 base pairs with $64.55 \%$ GC content, 56 CDS, and includes a plasmid stability gene (parA), transposases (Tn552, Tn554), and the insertion sequence IS6210. The second plasmid has $100 \%$ identity and

Table 3 Assembly Statistics

\begin{tabular}{|c|c|c|c|c|c|c|c|c|c|c|}
\hline & Sample & $\begin{array}{l}\text { Length } \\
\text { (bp) }\end{array}$ & Contigs & $\begin{array}{l}\text { N50 } \\
\text { (Mbp) }\end{array}$ & $\% G C$ & Coverage & $\begin{array}{l}\text { Circularized, complete } \\
\text { chromosome }\end{array}$ & Plasmids & $\begin{array}{l}\text { Completeness/ } \\
\text { Contamination }^{\text {b }}\end{array}$ & $\begin{array}{l}\text { Consistency, } \\
\text { Coarse/Fine }\end{array}$ \\
\hline \multirow{3}{*}{$\begin{array}{l}\text { Method } \\
1\end{array}$} & CHOP101034 & $5,440,506$ & 8 & 4.46 & 68.9 & $102.3 x$ & No & 2 & $100 / 2.5$ & $98.7 / 96.5$ \\
\hline & CHOP101115 & $5,391,592$ & 7 & 4.04 & 69.0 & $85.2 x$ & No & 2 & $100 / 5.7$ & $98.7 / 96.6$ \\
\hline & CHOP101174 & $5,171,418$ & 3 & 5.11 & 69.1 & $170.3 x$ & $Y_{e s}{ }^{a}$ & 2 & $100 / 1.7$ & $98.2 / 95.5$ \\
\hline \multirow{3}{*}{$\begin{array}{l}\text { Method } \\
5\end{array}$} & CHOP101034 & $5,368,111$ & 3 & 5.30 & 69.9 & $83.1 x$ & $Y_{e s}^{a}$ & 1 & 100/0.9 & $98.5 / 97.3^{*}$ \\
\hline & CHOP101115 & $5,393,712$ & 5 & 5.25 & 68.9 & $97.3 x$ & Yes & 2 & $100 / 0.9$ & $98.8 / 97.5^{*}$ \\
\hline & CHOP101174 & $5,130,681$ & 3 & 5.10 & 69.2 & $75.7 x$ & $Y_{e s}{ }^{a}$ & 2 & 100/0.9 & $98.7 / 97.3^{*}$ \\
\hline
\end{tabular}

Comparison of genomes assembled from reads generated from Method 1 versus Method 5 sequencing runs showing generally more complete assemblies from Method 5, with all Method 5 genomes producing complete and circularized bacterial chromosomes, while only $1 / 3$ bacterial chromosomes by Method 1 being circular and complete. All listed parameters were evaluated for statistical significance between the two methods. Method $5 \mathrm{genomes}$ had significantly higher fine consistency scores than Method 1 genomes by unpaired t-test, $p=0.0310$

*Indicates significance with $p<0.05$

${ }^{a}$ Complete and circularized chromosome and plasmids without extra unintegrated plasmid contigs

${ }^{b}$ Completeness is the percentage of genes with universal roles represented in the genome; Contamination approximates the percentage of the genome that is contaminated and is estimated by universal roles that are represented more than once in the genome [37]. 'Genome consistency estimates the percentage of universal roles expected to be present vs. absent (Coarse) in the genome and universal roles that are present in the exact number (Fine) as expected in the genome [37] 


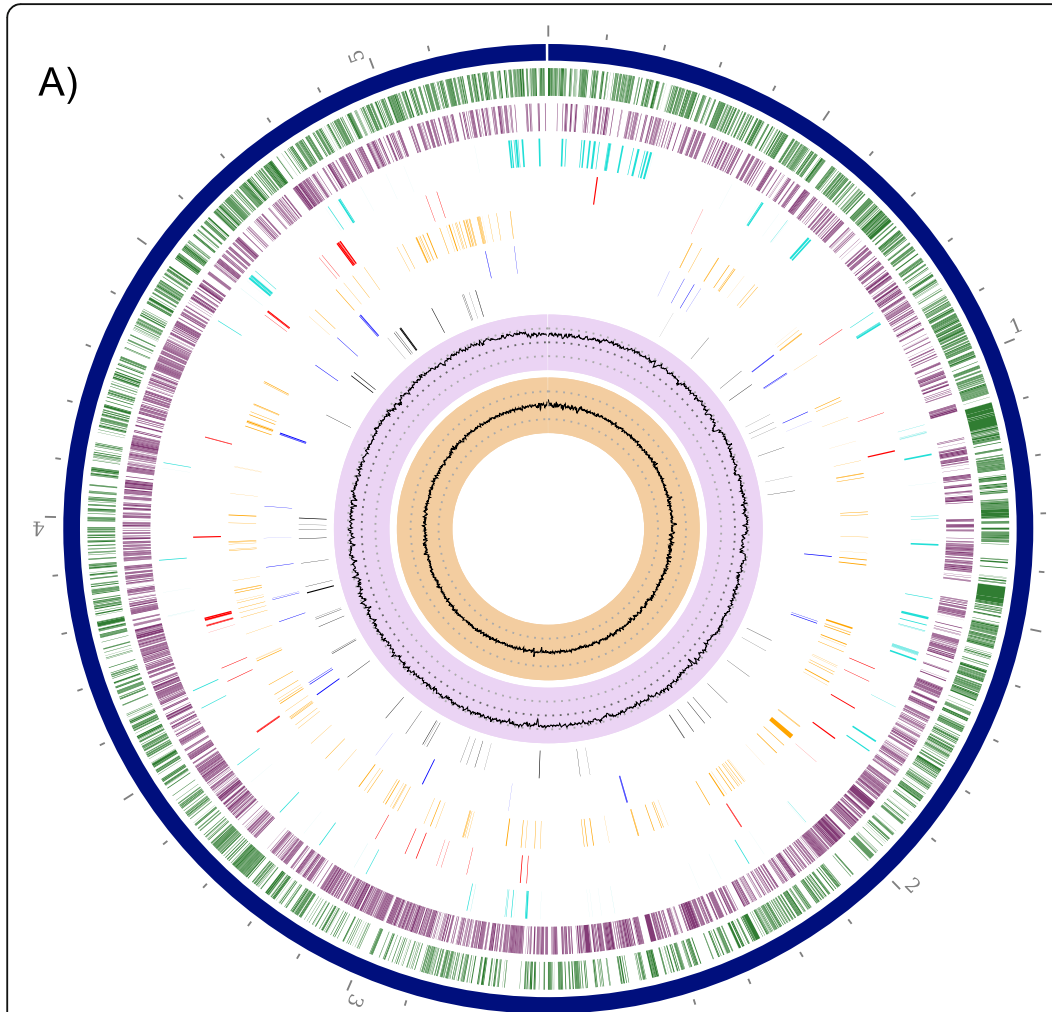

B)

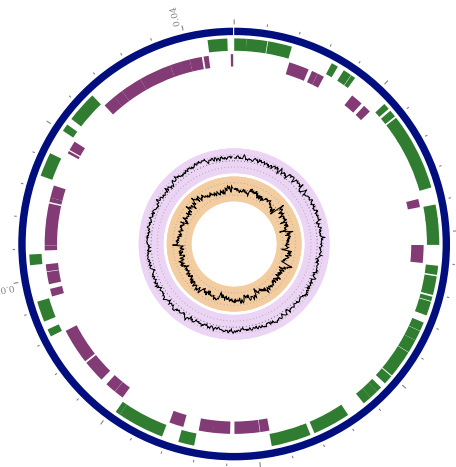

C)

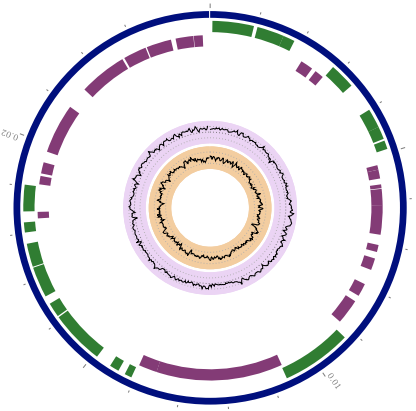

Fig. 3 Representative complete and circularized assembly. CHOP101034 bacterial 1 chromosome (a), pMARIA (b), and pFLAC0181_CHOP101 (c). Genome graphics by PATRIC [37], from outside in, position label (Mbp, grey), contigs (dark blue), CDS forward (green), CDS reverse (purple), non-CDS features (teal), antimicrobial resistance genes (red), virulence factor genes (orange), transporters (blue), drug targets (black), GC content (lavender), GC skew (peach)

$100 \%$ coverage to pFLAC0181. pFLAC0181_CHOP101 is 24,701 base pairs with $65.33 \%$ GC content, 31 CDS, and includes the plasmid stability gene parA, transmembrane proteins $(m m p L, m m p S)$, and a metal sensitive transcriptional repressor. CHOP101115 is composed of 5 contigs including a complete and circularized bacterial chromosome, pMARIA_2, pFLAC0181_CHOP101_2, and two linear contigs that identified most closely as partial plasmid sequences by NCBI blastn [38]. CHOP101115 is 5,393,712 base pairs with $68.94 \%$ GC content, 5,228 CDS, 97 repeat regions, 47 tRNAs, 3 rRNAs, and ONT coverage of 97.31x. CHOP101174 is comprised of a circularized bacterial chromosome, pMARIA_1, and pFLAC0181_CHOP101_ 1. CHOP101174 is $5,130,681$ base pairs with $69.18 \%$ GC content, 4,952 CDS, 76 repeat regions, 46 tRNAs, 3 rRNAs, and ONT coverage of 75.7x. The pMARIA plasmids were found to be highly similar to each other with percent identity ranging 96.0-99.95\%, and pFLAC0181 sequences ranged from 94.72-100\% identity by NCBI blastn alignments, with variations representing either sequencing error or natural variation and horizontal gene transfer occurring over time during chronic lung infection [38].

\section{Data accession}

The NTM Project at the Children's Hospital of Philadelphia, NCBI BioProject PRJNA532547, is available at https:// www.ncbi.nlm.nih.gov/sra/PRJNA532547. SRA reads are available for CHOP101034 (BioSample SAMN11403486), CHOP101115 (BioSample SAMN11403599), and CHOP101174 (BioSample SAMN11403589). CHOP101034 is represented by a complete, circularized bacterial chromosome (CP040247), pMARIA (CP040245), and pFLAC0181_ CHOP101 (CP040246). CHOP101115 is represented by a complete, circularized bacterial chromosome (CP040255), pMARIA_2 (CP040253), p_FLAC0181_CHOP101_2 (CP040254), and two plasmid fragments (CP040251, CP040252). CHOP101174 is represented by a complete, circularized bacterial chromosome (CP040250), pMARIA_ 1 (CP040249), and pFLAC0181_CHOP101_1 (CP040248).

\section{Conclusion}

Our protocol produced DNA of sufficient quantity and quality for long-read whole genome sequencing with the ONT MinION sequencer. To demonstrate direct comparisons to alternative methods, we completed DNA extraction with 6 variations of methodology with normalized starting bacterial pellet weights. Method 5 demonstrated 
superiority as the only method to provide appropriate DNA quality in all tested measurements without requiring any clean-up steps. Method 5 was characterized by early bead-beating in high-SDS concentration, gentle phenolbased extraction, and room temperature isopropanol precipitation. While Method 5 was the only method to use $\mathrm{NaCl}$ as the precipitation salt, later direct comparisons of $\mathrm{NaCl}$ versus $\mathrm{NaOAc}$ alone did not demonstrate any superiority of $\mathrm{NaCl}$. Thus, while either salt is appropriate, we recommend $\mathrm{NaCl}$ over $\mathrm{NaOAc}$ because it does not require $\mathrm{pH}$ titration. During development of our protocol we also trialed an alternative buffer, variable concentrations of lysozyme and proteinase $\mathrm{K}$, variable starting weights of bacterial pellets, extraction without beadbeating, and bead-beating with and without SDS, in addition to all the variables described in this manuscript (some of these variables are represented in Additional file 2: Table S1),

In comparison to the widely-used method by Käser et al. [32] that is useful for short-read sequencing, we noted improvements in the purity of DNA (260/230) with modifications of the composition of lysis buffer (Additional file 1), the timing of bead beating (early vs. late), the use of Phase Lock Gel ${ }^{\mathrm{Tm}}$ tubes, and the use of room temperature isopropanol as opposed to cold 100\% ethanol. Others have shown improved DNA purity with isopropanol extractions compared to cold ethanol extractions with less salt carry-over, albeit at the expense of DNA yields [31,32]. While Method 1 and 3 gave more total DNA, neither reached a suitable 260/230 absorbance. Thus, our method sacrifices total DNA yield to achieve high DNA purity. Our optimized method was also highly reliable, yielding sufficient quality and quantity for ONT sequencing in all $38 \mathrm{MAC}$ and MABSC clinical isolates, regardless of mycobacterial species or starting $\mathrm{OD}_{600}$.

The trademark of the mycobacterial cell wall is its hardy, heavily lipophilic exterior. In addition, mycobacterial peptidoglycans are characterized by an oxidation modification rendering lysozyme less effective at cleaving the $\beta[1,4]$ linkages between $\mathrm{N}$-acetylmuramic acid and $\mathrm{N}$-acetyl-D-glucosamine residues [39]. Thus, it is no surprise that mechanical cell wall disruption is necessary for DNA extraction. We reasoned that early mechanical disruption allows the exterior mycolic acid cell wall and peptidoglycan layer to be broken down first, with subsequent enzymatic digestion with lysozyme and proteinase $\mathrm{K}$ to digest the remainder of the cell wall and expose its inner contents. In our preliminary trials, early mechanical disruption demonstrated superiority to late mechanical disruption. Although not achieving statistical significance in the head-to-head comparisons presented here, we have consistently noted increased shearing with late mechanical disruption, resulting in homogenously distributed smears of MW DNA on gel electrophoresis. In addition, we found that the early addition of high concentrations of SDS during early beat-beating was also independently superior to bead-beating without SDS (Additional file 2: Table S1). The detergent properties of SDS likely assist with mechanical lysis and may additionally protect exposed DNA from degradation.

The optimized method presented here is able to produce large amounts of very pure, high molecular weight DNA without extra clean-up steps. The avoidance of clean-up steps is essential because repeat precipitationbased and SPRI bead-based clean-up methods consistently result in the loss of large amounts of DNA. Thus, a single method that is able to produce highly pure DNA without clean-up is critical in cases where larger amounts of DNA are desired for long-read sequencing modalities. Additional modifications in DNA preparation that should be considered are size selection and library kit selection. Size selection was intentionally not completed to avoid introduction of variability that could potentially lead to bias in method comparison. However, size selection should be considered prior to library preparation as it may increase sequenced read lengths. Interestingly, we found that use of SPRI bead-based size selection (used in the 8-sample run) did not significantly improve read lengths when using the ONT 1D Rapid Barcoding Kit (SQK-RBK004), by unpaired t-tests (Additional file 3: Table S2). As this particular kit is transposase-based and introduces breaks in DNA during adapter annealing, we suggest that if longer reads are desired, the Ligation Sequencing Kit could be used to achieve even longer reads. In which case, we recommend SPRI-bead size selection prior to library preparation, as the ligation kit does not require fragmentation for adapter annealing (https://nanoporetech.com/products/kits) [40]. However, even using the rapid kit with induced fragmentation of DNA and no size selection, we were able to complete bacterial genomes with our ONT-based assemblies.

For the three genomes presented, we notice slight variability in genome length. The genomes constructed for the exact same isolate by Method 1 versus Method 5 vary in length by about $1 \%$. For example, CHOP101174 varies by $0.8 \%$ in length between the two sequencing methods and both methods produced closed genomes, however the measurements of consistency were higher and contamination lower in the Method 5 genome. While these variations may be a result of sequencing error, they may also be due to an inability to resolve repeat regions and join contigs across regions where sequences have lower quality. In the setting of high GC content genomes with large repeat regions, a larger (and more fragmented) genome likely reflects duplications in repeat regions that cannot be resolved. Differences in genome length between the three isolates may also be a 
result of natural variation over time. While the 3 isolates sequenced are essentially biological replicates and are from the same patient, they were obtained at different time points during a chronic lung infection, and thus, differences may also reflect adaptation, recombination, insertion, or deletion. Taken as a whole, while the genome quality metrics for Method 5 are only slightly improved compared to Method 1, we favor the length results obtained by Method 5 based on its marginally better quality and closure. In addition, the Method 5 extraction technique is simpler and does not require extra clean-up steps.

Long-read sequencing offers a much higher likelihood than short-read sequencing of producing complete (circularized) genomes. Complete genomes can be used to more readily identify critical genomic characteristics such as extrachromosomal elements, mosaicism/recombination tracks, large repeat regions, duplications, and inversions, all of which likely play critical roles in mycobacterial genomic evolution; these elements can introduce signals (e.g., horizontal gene transfer) that confound phylogenetic inference $[19,28,29,41,42]$. Therefore, studies aimed at understanding patterns of transmission and genomic evolution that rely heavily on phylogeny gain power and accuracy when they consider complete genome sequences. Completed genomes also provide optimal reference sequences for comparison of clonal relatives because they contain a more complete picture of genome content and organization.

Our optimized extraction protocol and ONT assembly pipeline presented here were both sufficient and efficient for genome closure at a fraction of the cost and time of other approaches. Undoubtedly, long-read assembled genomes are the way of the future, but regardless of new technologies for cheap and high-fidelity DNA sequencing, we remain at the mercy of the cell wall, and we will continue to be faced with the delicate challenge of mining unscathed DNA from a distinctly robust substrate. We expect that our finely-tuned extraction method will prove to be a valuable tool in the mycobacterial genomics field going forward.

\section{Methods}

\section{Bacterial growth}

Clinical isolates of NTM were grown from frozen stocks to Löwenstein-Jensen slants and sub-cultured to Middlebrook $7 \mathrm{H} 11$ plates. Single colonies from 7H11 plates were inoculated in Middlebrook 7H9 broth supplemented with $10 \%$ Oleic Albumin Dextrose Catalase (OADC) and incubated at $37^{\circ} \mathrm{C}$ shaking for up to 2 weeks $\left(\mathrm{OD}_{600}>0.700\right)$. Bacterial cultures were pelleted and stored at $-20^{\circ} \mathrm{C}$ until the time of extraction. Initial comparative analyses of methods were done on MAC isolates, while the method validation was completed on both MAC and MABSC isolates.

\section{DNA extraction method optimization and validation}

The following extraction protocol described is our optimized method, "Method 5." Alterations in Method 5 for comparison are described in Table 1 . Method 3 is similar to a standard protocol as described by Käser et al., with the only difference being the composition of the lysis buffer [32]. The comprehensive protocol of Method 5 (optimized method) with thorough descriptions of each step and reagent recipes is provided in Additional file 1.

\section{Sample preparation}

Bacterial pellets were resuspended and washed in $350 \mu \mathrm{L}$ of $1 \mathrm{X}$ phosphate-buffered saline (PBS) using $2 \mathrm{~mL}$ microcentrifuge tubes. Due to variability in starting weights between bacterial isolate cultures, and for the purposes of comparing extraction methods, all weights were normalized after a second PBS wash and "washed weights" were recorded. The samples were heat-inactivated for $60 \mathrm{~min}$ at $95^{\circ} \mathrm{C}$, pelleted, and supernatant discarded.

\section{"Early" mechanical disruption in SDS followed by enzymatic digestion}

Bacterial pellets were resuspended in $400 \mu \mathrm{L}$ of lysis buffer and $100 \mu \mathrm{L}$ of $20 \%$ SDS. Samples were homogenized with glass beads (four 30-scycles, maximum setting, Fisher Scientific vortex mixer, MoBio adapter) (150 mg glass beads, 0.1-mm diameter, Research Products International). Subsequently, all vortexing was avoided. Cell walls were additionally lysed in lysozyme (final concentration $10 \mathrm{mg} / \mathrm{mL}$ ) for $1 \mathrm{~h}$ at $37^{\circ} \mathrm{C}$. Proteinase $\mathrm{K}$ (final concentration $200 \mu \mathrm{g} / \mathrm{mL}$ ) was added and samples were incubated at $37^{\circ} \mathrm{C}$ for $90 \mathrm{~min}$ with mixing by turning end-over-end by hand to create a homogenous suspension every $30 \mathrm{~min}$. The lysates were centrifuged $(2,000$ rcf for $10 \mathrm{~min}$ followed by $18,000 \mathrm{rcf}$ for $2 \mathrm{~min}$ ) and supernatants transferred to $2 \mathrm{~mL} 5$ Prime Light Phase Lock $\mathrm{Gel}^{\mathrm{Tw}}$ (PLG, QuantaBio) microcentrifuge tubes. Variables tested included enzymatic digestion prior to mechanical disruption (Methods 2,3) and the amount of matrix material (Method 4).

\section{Phenol:chloroform:isoamyl alcohol extraction}

To extract DNA, $500 \mu \mathrm{L}$ of phenol:chloroform:isoamyl alcohol (25:24:1, Tris-saturated, $\mathrm{pH}$ 8.0) was added to the PLG tubes. The tubes were rotated on a HulaMixer (ThermoFisher Scientific, United States) at $20 \mathrm{rpm}$ for $20 \mathrm{~min}$ and then centrifuged (2,000 $\mathrm{rcf}$ for $10 \mathrm{~min})$. The DNA-containing aqueous layer was transferred to a new $2 \mathrm{~mL}$ microcentrifuge tube with care to not aspirate the gel layer. Chloroform:isoamyl alcohol (24:1, Tris- 
saturated) without phenol was tested as a variable (Methods 1, 2, 4, 6, 7).

\section{Isopropanol precipitation}

For DNA precipitation, 1/10 volume of $5 \mathrm{M}$ sodium chloride $(\sim 20-45 \mu \mathrm{L})$ and 1 volume of room temperature isopropanol $(\sim 200-450 \mu \mathrm{L})$ was added to the samples. DNA was allowed to precipitate overnight at room temperature. The samples were then centrifuged (18,000 rcf for $30 \mathrm{~min}$ at $22{ }^{\circ} \mathrm{C}$, to avoid heating), washed with $700 \mu \mathrm{L} 70 \%$ ethanol $\left(18,000 \mathrm{rcf}\right.$ for $10 \mathrm{~min}$ at $\left.22^{\circ} \mathrm{C}\right)$, and the supernatant carefully discarded, with repeat of washing steps 3 times. The samples were air-dried at room temperature with lids open for $15 \mathrm{~min}$, resuspended in $100 \mu \mathrm{L}$ of Tris-Cl elution buffer (Qiagen, Germany), eluted overnight at room temperature on a nutator $(24 \mathrm{rpm}$ fixed speed, Fisherbrand $\left.^{\mathrm{Tx}}\right)$; nutator use is optional and only theoretically assists elution. DNA was stored at $4{ }^{\circ} \mathrm{C}$. Variables tested during precipitation include use of cold $100 \%$ ethanol (Methods 3, 6) and use of an alternative salt (Methods 1$4,6,7)$.

\section{DNA extraction method validation}

Clinical samples of MAC $(n=12)$ and MABSC $(n=26)$ were obtained from the clinical microbiology lab at the Children's Hospital of Philadelphia as frozen stocks collected from clinical isolates between 2011 and 2018. Frozen stocks were streaked to $7 \mathrm{H} 11$ plates and incubated at $37^{\circ} \mathrm{C}$ for approximately $2-6$ weeks. Single colonies were grown in $4 \mathrm{~mL} 7 \mathrm{H} 9$ plus $10 \%$ OADC shaking at $37^{\circ} \mathrm{C}$ to $\mathrm{OD}_{600}>0.500$, pelleted, and pellets frozen at $20^{\circ} \mathrm{C}$ until the time of extraction. DNA was extracted using Method 5 as described above.

\section{Quality measures}

DNA was heated to $55-65^{\circ} \mathrm{C}$ prior to quality assessment to ensure homogeneity of DNA per quality measurement guidelines [43]. DNA purity was assessed with NanoDrop 2000 UV-Vis Spectrophotometer (ThermoFisher Scientific, United States) by measurements of 260/280 to detect protein contamination and 260/230 to detect contamination by solvents and salts. DNA concentrations were measured with Qubit ${ }^{\circ}$ 2.0 Fluorometer dsDNA BR Assay (ThermoFisher Scientific, United States). Gel electrophoresis $(0.6 \%$ agarose ethidium bromide gel, $40 \mathrm{~V}$ for $1.5-2 \mathrm{~h}$ ) estimated MWs and shearing, which was confirmed by 4200 TapeStation system bioanalyzer (Agilient, United States) in selected samples.

\section{ONT whole genome sequencing and genome construction DNA preparation and sequencing}

Three MAC clinical isolates (CHOP101034, CHOP101115, and CHOP101174) were grown as described above and extracted each by Method 1 and by Method 5 .
Method 1 was chosen for comparative analyses to Method 5 as it had the highest total amount of DNA. Due to inadequate $260 / 230$ of samples extracted by Method 1, these extracts required "clean-up", which we completed by re-eluting in $100 \mu \mathrm{L}$ of elution buffer and repeating isopropanol precipitation 2-3 times prior to achieving adequate quality measurements for use in the ONT MinION. DNA from Method $1(n=3)$ and Method $5(n=3)$ was prepared for WGS with the Rapid Barcoding Kit (SQK-RBK-004, version: RBK_9054_v2_ revD_23Jan2018) with starting DNA with $400 \mathrm{ng}$ per sample barcode, $260 / 280$ of $\sim 1.8$, and $260 / 230$ of 2.0-2.2 (with optional Solid Phase Reversible Immobilization bead step excluded due to barcoding of under 4 samples), and libraries sequenced on the ONT FLO-MIN107 (R9.4) flow cell. DNA from each of the 3 isolates was additionally sequenced by Illumina HiSeq 2500 using the Nextera XT library preparation kit (Illumina, US). Illumina short reads were demultiplexed with DNAbc and trimmed with Trim Galore! with default settings $[44,45]$.

\section{Genome completion}

Raw ONT fast5 output was basecalled with Guppy (ONT, v2.3.1 + 1b9405b), trimmed and demultiplexed with Porechop (-b, barcoding mode on, v0.2.4) [46], and fastq reads filtered with Filtlong (--keep_percent 90 --min_length 1000 --target_bases 500,00,000 --trim --split 500, without an external quality reference, v0.2.0) [47]. Genome assemblies were constructed using the tool Unicycler (default settings, --mode normal, v0.4.8-beta) [48], which was created specifically for utilization of ONT long-reads in the assembly of bacterial genomes. With input of basecalled, filtered fastq long-reads, Unicycler constructs a miniasm [49] assembly graph, polishes with Racon [37], and produces assembly graphs that can be viewed in Bandage [48, 50]. We additionally circularized with Circlator (circlator all, v1.5.5) [51] using input of the Unicycler-assembled genome and ONT long-reads corrected by Canu (canu -correct, genomeSize $=5.2 \mathrm{~m}$, errorRate $=0.144$, - nanopore-raw, v1.8) [52]. Post-Circlator assemblies were polished with Illumina short-reads using Pilon (--genome --frags, v.1.22) to improve genome quality [53]. Final assemblies were annotated in PATRIC (v3.5.34, patricbrc.org/) [54]. Assemblies were identified by the Kraken2 taxonomic sequence classification system (kraken2 --db bacteria, default settings, v2.0.7-beta) [55]. Coverage was calculated as an average of basecalled and trimmed fastq reads to the corresponding genome assembly using minimap2 alignment and taking the average read coverage of the samtools depth output (minimap2 -ax map-ont; samtools sort; samtools index; samtools depth) $[49,56]$. 


\section{Statistical analyses}

Statistical analyses were completed in Prism 7.0d for Mac OS X (GraphPad Software, La Jolla California USA, www.graphpad.com). Quality and quantity measurements of DNA extractions were compared by one-way ANOVA with post-hoc Tukey's multiple comparison tests and unpaired t-tests when appropriate. Linear regression line-ofbest-fit analyses were completed to investigate the contribution of starting $\mathrm{OD}_{600}$ on extraction outcome measures. ONT read qualities were assessed with NanoPack tools [57], assemblies received quality assessments in QUAST (v.5.0.2, quast.bioinf.spbau.ru) [58], and quality measurements for both reads and assemblies were compared with paired and unpaired t-tests when appropriate. Quality measurements of the genomes were additionally conducted by analyzing presence/absence and frequency of universal genes represented in the genomes in PATRIC, which were compared by unpaired t-tests [54].

\section{Supplementary information}

Supplementary information accompanies this paper at https://doi.org/10. 1186/s12864-019-6134-y.

Additional file 1. Protocol for DNA Extraction of Nontuberculous Mycobacteria for Long-Read Whole Genome Sequencing.

Additional file 2: Table S1. Previous grid experiments. Here we provide an outline of previous extractions, variations in protocols, and extraction quantity and quality measurements that led to the development of our optimized method.

Additional file 3: Table S2. DNA extraction and ONT sequencing statistics for additional 8 samples. Eight NTM isolates from 8 different patients were extracted by our optimized method and sequenced on the ONT MinION. Notably, SPRI bead size selection was completed on all samples and input DNA was increased to $1000 \mu \mathrm{g}$ for this sequencing run. The ONT Rapid Barcoding Kit was again used. There were no significant differences between MAC and MAB strains when comparing any of the listed statistics (unpaired, parametric t-test), nor were there any significant differences in any listed statistics when compared to the previous ONT sequencing run without size selection. Two outliers were observed with higher total reads and total bases (CHOP118112 and CHOP1500921), which may reflect variability of adapter annealing during barcoding preparation. Decreased coverage per barcode is observed compared to the ONT MinION runs with fewer barcoded samples, as expected.

Additional file 4: Figure S1. Sequencing statistics produced by NanoPack Tools [56] demonstrating violin plots of (A) read lengths over time, (B) quality over time, (C) log lengths of reads by barcode, and dot plots of (D) read lengths versus average read quality for each ONT MinION run.

Additional file 5: Table S4. Strain List of extractions by optimized method (Method 5). Listed below are the source, date collected, and extraction quality measures for all isolates described that were extracted by Method 5 .

Additional file 6: Table S3. Short-read Statistics. Short-reads were sequenced by Illumina HiSeq 2500 after Nextera XT library preparation, demultiplexed with DNAbc, and trimmed with Trim Galore! $[43,44]$. No significant differences were found between the sequenced samples with regard to total reads or quality by Phred score (unpaired t-tests).

\section{Abbreviations}

CDS: Coding sequences; CF: Cystic fibrosis; COPD: Chronic obstructive pulmonary disease; MABSC: Mycobacterium abscessus complex;
MAC: Mycobacterium avium complex; NTM: Nontuberculous mycobacteria; ONT: Oxford Nanopore Technologies; PacBIO: Pacific Biosciences;

SDS: Sodium dodecyl sulfate; SEM: Standard error of the mean; SMRT: Single Molecule Real Time; WGS: Whole genome sequencing

\section{Acknowledgements}

There are no acknowledgements.

\section{Authors' contributions}

JMB and PJP made substantial contributions to the conception and design, analysis, interpretation of data, and all results and conclusions drawn from this work. JMB and PJP have substantially contributed to the drafting and revision of the work and have approved the submitted version. JMB and PJP agree to be personally accountable for their own contributions and to ensure that questions related to the accuracy or integrity of any part of the work, even ones in which the author was not personally involved, are appropriately investigated, resolved, and the resolution documented in the literature.

\section{Funding}

This research was supported by the Cystic Fibrosis Foundation (BOUSO17B0) The funders had no role in study design, data collection and interpretation, or the decision to submit the work for publication.

\section{Availability of data and materials}

The NTM Project at the Children's Hospital of Philadelphia, NCBI BioProject PRJNA532547, is available at https://www.ncbi.nlm.nih.gov/sra/PRJNA532547. Please see "Data Accession" in the Results section for identification of reads and genomes associated with specific NCBI BioSamples.

\section{Ethics approval and consent to participate}

The research presented here was approved by the Children's Hospital of Philadelphia Institutional Review Board (CHOP IRB \#17-014648) for the retrospective collection and sequencing of microbiologic specimens stored in the CHOP Clinical Microbiology Laboratory. The IRB-approved protocol above allows for waived consent for all patients who submitted respiratory samples used in this project.

\section{Consent for publication}

Not applicable.

\section{Competing interests}

The authors declare that they have no competing interests.

\section{Author details}

${ }^{1}$ Division of Pulmonary Medicine, Children's Hospital of Philadelphia, Philadelphia, PA, USA. 'Division of Infectious Diseases, Children's Hospital of Philadelphia, Philadelphia, PA, USA. ${ }^{3}$ Perelman School of Medicine, University of Pennsylvania, Philadelphia, PA, USA. ${ }^{4}$ Sackler Institute for Comparative Genomics, American Museum of Natural History, New York, NY, USA.

Received: 4 June 2019 Accepted: 23 September 2019

Published online: 30 October 2019

\section{References}

1. Henkle E, Winthrop KL. Nontuberculous mycobacteria infections in immunosuppressed hosts. Clin Chest Med. 2015;36:91-9.

2. Prevots DR, Marras TK. Epidemiology of human pulmonary infection with nontuberculous mycobacteria: a review. Clin Chest Med. 2015;36:13-34.

3. Falkinham JO 3rd. Current epidemiologic trends of the Nontuberculous mycobacteria (NTM). Curr Environ Health Rep. 2016;3:161-7.

4. Szymanski EP, Leung JM, Fowler CJ, Haney C, Hsu AP, Chen F, Duggal P, Oler AJ, McCormack R, Podack E, Drummond RA, Lionakis MS, Browne SK, Prevots DR, Knowles M, Cutting G, Liu X, Devine SE, Fraser CM, Tettelin H, Olivier KN, Holland SM. Pulmonary Nontuberculous mycobacterial infection. A multisystem, multigenic disease. Am J Respir Crit Care Med. 2015;192:618-28.

5. Griffith DE, Aksamit T, Brown-Elliott BA, Catanzaro A, Daley C, Gordin F, Holland SM, Horsburgh R, Huitt G, lademarco MF, Iseman M, Olivier K, Ruoss S, von Reyn CF, Wallace RJ, Winthrop K, Subcommittee AMD, Society AT, America IDSo. An official ATS/IDSA statement: diagnosis, treatment, and 
prevention of nontuberculous mycobacterial diseases. Am J Respir Crit Care Med. 2007;175:367-416.

6. Martiniano SL, Davidson RM, Nick JA. Nontuberculous mycobacteria in cystic fibrosis: updates and the path forward. Pediatr Pulmonol. 2017;52:S29-36.

7. Viviani L, Harrison MJ, Zolin A, Haworth CS, Floto RA. Epidemiology of nontuberculous mycobacteria (NTM) amongst individuals with cystic fibrosis (CF). J Cyst Fibros. 2016. https://doi.org/10.1016/j.jcf.2016.03.002.

8. Davidson RM, Hasan NA, Reynolds PR, Totten S, Garcia B, Levin A, Ramamoorthy P, Heifets L, Daley CL, Strong M. Genome sequencing of Mycobacterium abscessus isolates from patients in the United States and comparisons to globally diverse clinical strains. J Clin Microbiol. 2014;52: 3573-82.

9. Park IK, Olivier KN. Nontuberculous mycobacteria in cystic fibrosis and noncystic fibrosis bronchiectasis. Semin Respir Crit Care Med. 2015;36:217-24.

10. Kreutzfeldt KM, McAdam PR, Claxton P, Holmes A, Seagar AL, Laurenson IF, Fitzgerald JR. Molecular longitudinal tracking of Mycobacterium abscessus spp. during chronic infection of the human lung. PLoS One. 2013;8:e63237.

11. Olsen RJ, Long SW, Musser JM. Bacterial genomics in infectious disease and the clinical pathology laboratory. Arch Pathol Lab Med. 2012;136:1414-22.

12. Bentley SD, Parkhill J. Genomic perspectives on the evolution and spread of bacterial pathogens. Proc Biol Sci. 2015;282:20150488.

13. Firth C, Lipkin WI. The genomics of emerging pathogens. Annu Rev Genomics Hum Genet. 2013;14:281-300.

14. Cao MD, Nguyen SH, Ganesamoorthy D, Elliott AG, Cooper MA, Coin LJ. Scaffolding and completing genome assemblies in real-time with nanopore sequencing. Nat Commun. 2017;8:14515.

15. Sharma P, Gupta SK, Rolain JM. Whole genome sequencing of bacteria in cystic fibrosis as a model for bacterial genome adaptation and evolution. Expert Rev Anti-Infect Ther. 2014;12:343-55.

16. Bottai D, Stinear TP, Supply P, Brosch R. Mycobacterial Pathogenomics and evolution. Microbiol Spectr. 2014;2:MGM2-0025-2013.

17. Brown-Elliott BA, Philley JV. Rapidly growing mycobacteria. Microbiol Spectr. 2017:5:1.

18. Claeys TA, Robinson RT. The many lives of nontuberculous mycobacteria. J Bacteriol. 2018. https://doi.org/10.1128/jb.00739-17.

19. Sapriel G, Konjek J, Orgeur M, Bouri L, Frezal L, Roux AL, Dumas E, Brosch R, Bouchier C, Brisse S, Vandenbogaert M, Thiberge JM, Caro V, Ngeow YF, Tan JL, Herrmann JL, Gaillard JL, Heym B, Wirth T. Genome-wide mosaicism within Mycobacterium abscessus: evolutionary and epidemiological implications. BMC Genomics. 2016;17:118.

20. Bryant JM, Grogono DM, Rodriguez-Rincon D, Everall I, Brown KP, Moreno P, Verma D, Hill E, Drijkoningen J, Gilligan P, Esther CR, Noone PG, Giddings O, Bell SC, Thomson R, Wainwright CE, Coulter C, Pandey S, Wood ME, Stockwell RE, Ramsay KA, Sherrard LJ, Kidd TJ, Jabbour N, Johnson GR, Knibbs LD, Morawska L, Sly PD, Jones A, Bilton D, Laurenson I, Ruddy M, Bourke S, Bowler IC, Chapman SJ, Clayton A, Cullen M, Daniels T, Dempsey O, Denton M, Desai M, Drew RJ, Edenborough F, Evans J, Folb J, Humphrey $H$, Isalska B, Jensen-Fangel $S$, Jonsson B, Jones AM, et al. Emergence and spread of a human-transmissible multidrug-resistant nontuberculous mycobacterium. Science. 2016;354:751-7.

21. Miranda-CasoLuengo AA, Staunton PM, Dinan AM, Lohan AJ, Loftus BJ. Functional characterization of the Mycobacterium abscessus genome coupled with condition specific transcriptomics reveals conserved molecular strategies for host adaptation and persistence. BMC Genomics. 2016;17:553.

22. Davidson RM, Hasan NA, de Moura VC, Duarte RS, Jackson M, Strong M. Phylogenomics of Brazilian epidemic isolates of Mycobacterium abscessus subsp. bolletii reveals relationships of global outbreak strains. Infect Genet Evol. 2013;20:292-7.

23. Gupta RS, Lo B, Son J. Phylogenomics and comparative genomic studies robustly support division of the genus Mycobacterium into an emended genus Mycobacterium and four novel genera. Front Microbiol. 2018;9:67.

24. Bryant JM, Grogono DM, Greaves D, Foweraker J, Roddick I, Inns T, Reacher M, Haworth CS, Curran MD, Harris SR, Peacock SJ, Parkhill J, Floto RA. Whole-genome sequencing to identify transmission of Mycobacterium abscessus between patients with cystic fibrosis: a retrospective cohort study. Lancet. 2013;381:1551-60.

25. Land M, Hauser L, Jun SR, Nookaew I, Leuze MR, Ahn TH, Karpinets T, Lund O, Kora G, Wassenaar T, Poudel S, Ussery DW. Insights from 20 years of bacterial genome sequencing. Funct Integr Genomics. 2015;15:141-61.

26. Nakano K, Shiroma A, Shimoji M, Tamotsu H, Ashimine N, Ohki S, Shinzato M, Minami M, Nakanishi T, Teruya K, Satou K, Hirano T. Advantages of genome sequencing by long-read sequencer using SMRT technology in medical area. Hum Cell. 2017;30:149-61.

27. Wick RR, Judd LM, Gorrie CL, Holt KE. Completing bacterial genome assemblies with multiplex MinION sequencing. Microb Genom. 2017;3:e000132.

28. Timms VJ, Hassan KA, Mitchell HM, Neilan BA. Comparative genomics between human and animal associated subspecies of the Mycobacterium avium complex: a basis for pathogenicity. BMC Genomics. 2015;16:695.

29. Bainomugisa A, Duarte T, Lavu E, Pandey S, Coulter C, Marais BJ, Coin LM. A complete high-quality MinION nanopore assembly of an extensively drugresistant Mycobacterium tuberculosis Beijing lineage strain identifies novel variation in repetitive PE/PPE gene regions. Microb Genom. 2018;4.

30. Rhoads A, Au KF. PacBio sequencing and its applications. Genomics Proteomics Bioinformatics. 2015;13:278-89.

31. Käser M, Ruf MT, Hauser J, Marsollier L, Pluschke G. Optimized method for preparation of DNA from pathogenic and environmental mycobacteria. Appl Environ Microbiol. 2009;75:414-8.

32. Kaser M, Ruf MT, Hauser J, Pluschke G. Optimized DNA preparation from mycobacteria. Cold Spring Harb Protoc. 2010;2010:pdb prot5408.

33. Caverly LJ, Carmody LA, Haig S-J, Kotlarz N, Kalikin LM, Raskin L, LiPuma JJ. Culture-independent identification of Nontuberculous mycobacteria in cystic fibrosis respiratory samples. PLoS One. 2016;11:e0153876.

34. Votintseva AA, Bradley P, Pankhurst L, Del Ojo EC, Loose M, Nilgiriwala K, Chatterjee A, Smith EG, Sanderson N, Walker TM, Morgan MR, Wyllie DH, Walker AS, Peto TEA, Crook DW, Iqbal Z. Same-day diagnostic and surveillance data for tuberculosis via whole-genome sequencing of direct respiratory samples. J Clin Microbiol. 2017;55:1285-98.

35. Votintseva AA, Pankhurst LJ, Anson LW, Morgan MR, Gascoyne-Binzi D, Walker TM, Quan TP, Wyllie DH, Del Ojo EC, Wilcox M, Walker AS, Peto TE, Crook DW. Mycobacterial DNA extraction for whole-genome sequencing from early positive liquid (MGIT) cultures. J Clin Microbiol. 2015;53:1137-43.

36. Quick J. 2018. Ultra-long read sequencing protocol for RAD004, on protocols.lo. doi:https://doi.org/10.17504/protocols.io.mrxc57n. Accessed 24 Feb 2018.

37. Vaser R, Sovic I, Nagarajan N, Sikic M. Fast and accurate de novo genome assembly from long uncorrected reads. Genome Res. 2017;27:737-46.

38. Thomas Madden. 2002. The BLAST sequence analysis tool. In J M, J O (ed), The NCBI handbook. National Center for Biotechnology Information, Bethesda.

39. Jankute M, Cox JA, Harrison J, Besra GS. Assembly of the mycobacterial Cell Wall. Annu Rev Microbiol. 2015;69:405-23.

40. Tyler AD, Mataseje L, Urfano CJ, Schmidt L, Antonation KS, Mulvey MR, Corbett CR. Evaluation of Oxford Nanopore's MinION sequencing device for microbial whole genome sequencing applications. Sci Rep. 2018:8:10931.

41. Mukhopadhyay S, Balaji KN. The PE and PPE proteins of Mycobacterium tuberculosis. Tuberculosis (Edinb). 2011;91:441-7.

42. Uchiya Kl, Tomida S, Nakagawa T, Asahi S, Nikai T, Ogawa K. Comparative genome analyses of Mycobacterium avium reveal genomic features of its subspecies and strains that cause progression of pulmonary disease. Sci Rep. 2017;7:39750.

43. Anonymous. NanoDrop one user guide, vol T044-TECHNICAL BULLETIN p15. Wilmington: Thermo Fisher Scientific; 2017.

44. PennCHOPMicrobiomeProgram. DNAbc. https://github.com/ PennChopMicrobiomeProgram/dnabc/. Accessed 28 Sept 2018.

45. Kreuger F. Trim Galore. http://www.bioinformatics.babraham.ac.uk/projects/ trim_galore/. Accessed 28 Sept 2018.

46. Wick RR. 2017. Porechop. https://github.com/rrwick/Porechop/. Accessed 2 Feb 2019.

47. Wick RR. 2018. Filtlong. https://github.com/rrwick/Filtlong/. Accessed 2 Feb 2019.

48. Wick RR, Judd LM, Gorrie CL, Holt KE. Unicycler: resolving bacterial genome assemblies from short and long sequencing reads. PLoS Comput Biol. 2017; 13:e1005595.

49. Li H. Minimap and miniasm: fast mapping and de novo assembly for noisy long sequences. Bioinformatics. 2016:32:2103-10.

50. Wick RR, Schultz MB, Zobel J, Holt KE. Bandage: interactive visualization of de novo genome assemblies. Bioinformatics. 2015;31:3350-2.

51. Hunt M, Silva ND, Otto TD, Parkhill J, Keane JA, Harris SR. Circlator: automated circularization of genome assemblies using long sequencing reads. Genome Biol. 2015;16:294.

52. Koren S, Walenz BP, Berlin K, Miller JR, Bergman NH, Phillippy AM. Canu: scalable and accurate long-read assembly via adaptive k-mer weighting and repeat separation. Genome Res. 2017;27:722-36. 
53. Walker BJ, Abeel T, Shea T, Priest M, Abouelliel A, Sakthikumar S, Cuomo CA, Zeng Q, Wortman J, Young SK, Earl AM. Pilon: an integrated tool for comprehensive microbial variant detection and genome assembly improvement. PLoS One. 2014;9:e112963.

54. Wattam AR, Abraham D, Dalay O, Disz TL, Driscoll T, Gabbard JL, Gillespie JJ, Gough R, Hix D, Kenyon R, Machi D, Mao C, Nordberg EK, Olson R, Overbeek R, Pusch GD, Shukla M, Schulman J, Stevens RL, Sullivan DE, Vonstein V, Warren A, Will R, Wilson MJ, Yoo HS, Zhang C, Zhang Y, Sobral BW. PATRIC, the bacterial bioinformatics database and analysis resource. Nucleic Acids Res. 2014;42:D581-91.

55. Wood DE, Salzberg SL. Kraken: ultrafast metagenomic sequence classification using exact alignments. Genome Biol. 2014;15:R46.

56. Li H, Handsaker B, Wysoker A, Fennell T, Ruan J, Homer N, Marth G, Abecasis G, Durbin R. The sequence alignment/map format and SAMtools. Bioinformatics. 2009;25:2078-9.

57. De Coster W, D'Hert S, Schultz DT, Cruts M, Van Broeckhoven C. NanoPack: visualizing and processing long-read sequencing data. Bioinformatics. 2018; 34:2666-9.

58. Gurevich A, Saveliev V, Vyahhi N, Tesler G. QUAST: quality assessment tool for genome assemblies. Bioinformatics. 2013;29:1072-5.

\section{Publisher's Note}

Springer Nature remains neutral with regard to jurisdictional claims in published maps and institutional affiliations.

Ready to submit your research? Choose BMC and benefit from:

- fast, convenient online submission

- thorough peer review by experienced researchers in your field

- rapid publication on acceptance

- support for research data, including large and complex data types

- gold Open Access which fosters wider collaboration and increased citations

- maximum visibility for your research: over $100 \mathrm{M}$ website views per year

At $\mathrm{BMC}$, research is always in progress.

Learn more biomedcentral.com/submissions 